\title{
The Atomic Structure of Oxide Superlattices Revealed by Fine Electron Probes
}

A. B. Shah, ${ }^{1,2}$ Q. M. Ramasse, ${ }^{3}$ S. J. May, ${ }^{4}$ T. Santos, ${ }^{4}$ J. G. Wen, ${ }^{2}$ X. Zhai,${ }^{2,5}$ J. N. Eckstein, ${ }^{2,5}$ A. Bhattacharya, ${ }^{4,6}$ and J. M. Zuo. ${ }^{1,2}$

${ }^{1}$ Dept. of Mats. Sci. and Engineering, University of Illinois at Urbana-Champaign, Urbana, IL 61801

${ }^{2}$ F. Seitz Materials Research Lab, University of Illinois at Urbana-Champaign, Urbana, IL 61801

${ }^{3}$ Natl. Center for Electron Microscopy, Lawrence-Berkeley National Lab., Berkeley, CA 94720

${ }^{4}$ Materials Science Division, Argonne National Laboratory, Argonne, IL 60439

${ }^{5}$ Dept. of Physics, University of Illinois at Urbana-Champaign, Urbana, IL, 61801

${ }^{6}$ Center for Nanoscale Materials, Argonne National Laboratory, Argonne, IL 60439

Perovskite oxide superlattices have attracted much interest due to unique properties such as ferromagnetism and colossal magnetoresistence, and advances in growth techniques which can engineer layer-by-layer superlattices. One of critical issues of these superlattices is the accommodation of strain as films of slightly different lattice parameter are grown lattice-matched to the substrate and constrained by epitaxy. Strain and the associated atomic displacements often play an important role in the electronic structures of perovskites. Characterization of strain and their distribution are essential to understand the unique properties of those oxide superlattices $[1,2,3]$.

We use a combination of aberration-corrected (AC), high angle annular dark field (HAADF), scanning transmission electron microscopy (STEM) and electron nanodiffraction to characterize the atomic structure of oxide superlattices. AC-HAADF-STEM provides atomic resolution and the sensitivity to metal cations. Electron nanodiffraction is sensitive to both metal cations and oxygen. The contrast from annular dark field (ADF) STEM also can be used to distinguish between rough and sharp interfaces $[1,3]$. The new generation of AC-STEMs also can be used to acquire images with large field of views without suffering large sample drifts. For example, it is possible to acquire atomic-level images at field of views greater than $100 \mathrm{~nm}$ x $100 \mathrm{~nm}$. The Fourier transform of such images shows superlattice reflections and other weak reflections. This is a useful aid to diffraction since if we are able to acquire a large amount of pixels in the STEM image, we can directly determine from the image where weak spots spot in the Fourier spectrum originate. For example, we can distinguish the crystal structure of two films in a superlattice; such information is difficult to obtain by diffraction without inverting the diffraction patterns.

We have investigated several $\mathrm{LaMnO}_{3}-\mathrm{SrMnO}_{3}$ (LMO-SMO) superlattices grown on (001) $\mathrm{SrTiO}_{3}$ by molecular beam epitaxy. Figure 1 shows a STEM image that shows sharp and rough interfaces. The fast Fourier transform (FFT) of the image shows weak reflections from two orthorhombic domains of LMO, which are confirmed by nanoarea electron diffraction with a parallel beam. Using a mask of weak reflections in the FFT, we observe weak reflections and their distribution in the superlattice. Surprisingly, both domains are observed but some layers are dominated by one of the two domains. We have performed such studies for several similar samples of different layer thicknesses. When the $\mathrm{LaMnO}_{3}$ film was 6 unit cells in thickness or greater, the weak reflections were present in diffraction patterns, but when the $\mathrm{LaMnO}_{3}$ film was 4 unit cells or thinner, the weak reflections were absent. We will discuss the orthorhombic crystal structure and its significance which is present in the $\mathrm{LaMnO}_{3}$ films. 


\section{References:}

[1] S. J. May et al., Phys. Rev. B. 77 (2008) 174409.

[2] A. Ohtomo et al., Nature 419 (2002) 378.

[3] L. Fitting Kourkoutis et al., Microsc Microanal 14 (Suppl 2) (2008) 12.

[4] We acknowledge the support from the U.S. Department of Energy, Office of Basic Energy

Sciences under Contracts No. DE-AC02-06CH11357, No. DEFG02-01ER45923 and No. DEFG0291ER45439, the Fredrick Sietz Materials Research Laboratory at the University of Illinois, and the National Center for Electron Microscopy at Lawrence-Berkeley National Laboratory.
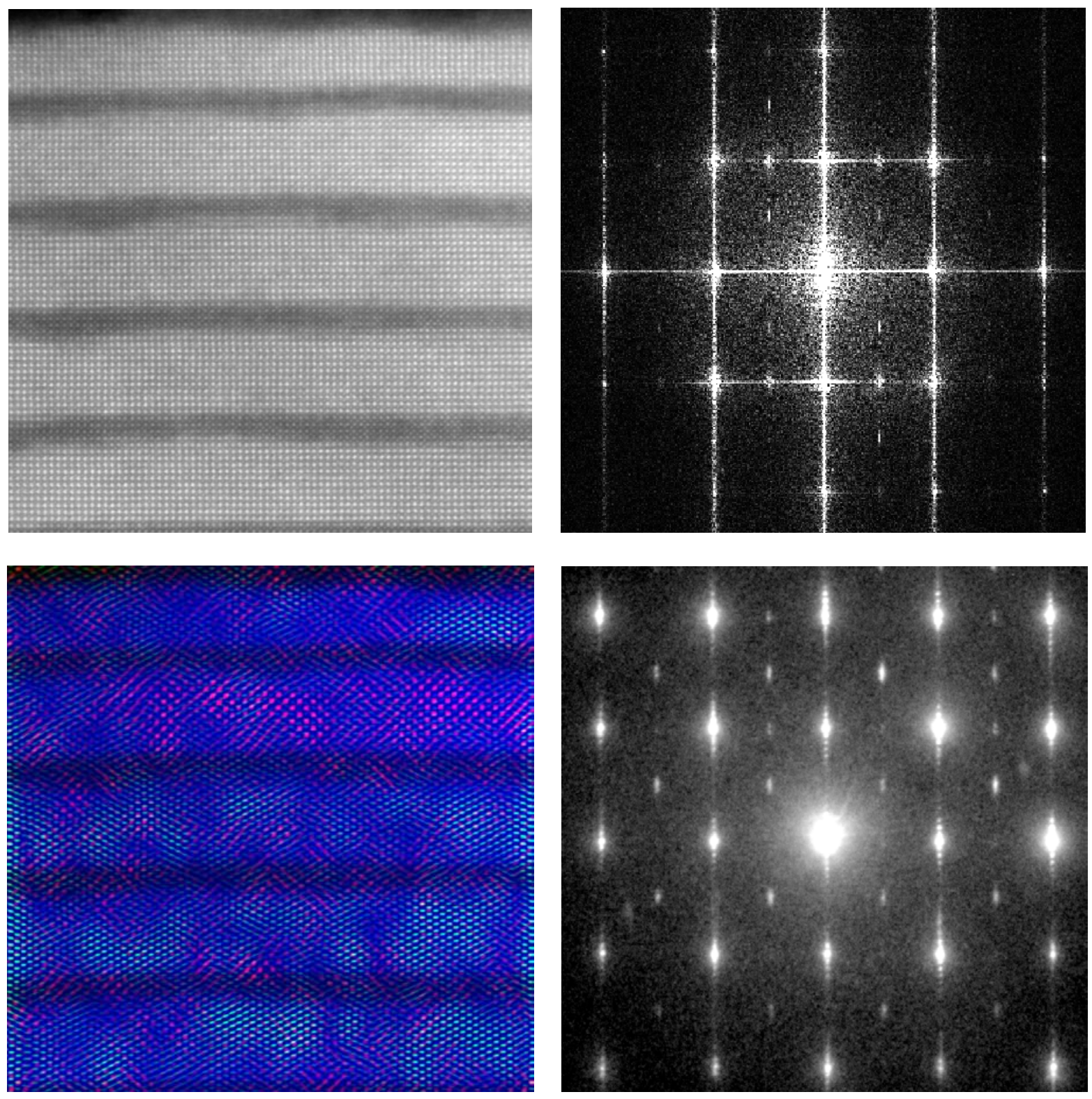

Figure 1: (a) ADF STEM image of a $\mathrm{LaMnO}_{3}-\mathrm{SrMnO}_{3}$ superlattice grown on $\mathrm{SrTiO}_{3}$. (b) FFT of the image in (a) shows weak reflections. (c) RGB image formed by overlayed STEM image and images from inverse FFTs of masked weak reflections. (d) Nanoarea electron diffraction pattern confirms the weak reflections and superlattice reflections. 